\title{
Colors and luminosities of the optical afterglows of the $\gamma$-ray bursts
}

\author{
V. Šimon ${ }^{1}$, R. Hudec ${ }^{1}$, G. Pizzichini ${ }^{2}$, and N. Masetti ${ }^{2}$ \\ 1 Astronomical Institute, Academy of Sciences of the Czech Republic, 25165 Ondřejov, Czech Republic \\ 2 Istituto Tecnologie e Studio delle Radiazioni Extraterrestri, CNR, via Gobetti 101, 40129 Bologna, Italy
}

Received 26 December 2000 / Accepted 16 August 2001

\begin{abstract}
Results of the study of the color indices and luminosities of 17 optical afterglows (OAs) of GRBs are presented. We show that the color variations during the decline of OAs (except for GRB000131) are relatively small during $t-T_{0}<10$ days and allow a comparison among them, even for the less densely sampled OAs. The colors in the observer frame, corrected for the Galactic reddening, concentrate at $(V-R)_{0}=0.40 \pm 0.13$, $(R-I)_{0}=0.46 \pm 0.18,(B-V)_{0}=0.47 \pm 0.17$. The color evolution of the OAs is negligible although their brightness declines by several magnitudes during the considered time interval. Such a strong concentration of the color indices also suggests that the intrinsic reddening (inside their host galaxies) must be quite similar and relatively small for all these events. The absolute brightness of OAs in the observer frame, corrected for the host galaxy, lies within $M_{R_{0}}=-26.5$ to -22.2 for $\left(t-T_{0}\right)_{\text {rest }}=0.25$ days. This spread of $M_{R_{0}}$ is not significantly influenced by the shifts of $\lambda$, caused by the different redshift $z$ of the respective OAs. The general decline rate of the OA sample considered here seems to be independent of the absolute optical brightness of the OA, measured at some $t-T_{0}$ identical for all OAs, and the light curves of all events are almost parallel, when corrected for the redshift-induced time dilation.
\end{abstract}

Key words. gamma rays: bursts - radiation mechanisms: non-thermal - plasmas - ISM: jets and outflows galaxies: ISM - galaxies: starburst

\section{Introduction}

The color indices of the optical afterglows (OAs) of the $\gamma$-ray bursts (GRB) are a powerful tool to use in the search for the common properties of these events. The OAs in the fireball model represent the stadium when the matter from the central engine, moving at the relativistic speed, interacts with the surrounding interstellar medium by means of external shocks (see Piran 1999 for review). The color indices of the OAs can be used as an important parameter reflecting the related physical processes. Besides the astrophysical analysis, the specific color indices of OAs give hope to resolving whether an optical transient event is related to a GRB even without available $\gamma$-ray detection (see also e.g. Hudec 2000; Rhoads 2001). The redshift $z$, known for some OAs, can help assess the role of the shifts of optical passbands with respect to the rest frame. The distance of OAs can be determined from their redshift $z$ enabling their absolute brightnesses to be calculated. The interrelations among the colors, luminosities and the decay rates of the OAs can thus be searched for. Moreover, we will show that the colors of the OAs enable us to put

Send offprint requests to: V. Šimon, e-mail: simon@asu.cas.cz stringent constraints on the properties of the local interstellar medium of the GRBs considered here.

The preliminary versions of this analysis were presented by Šimon et al. (2000a,b,c).

\section{Collection and analysis of the data}

This comprehensive analysis has made use of the data published in the journals, the GCN circulars $^{1}$ and in J. Greiner's web page ${ }^{2}$. The summary of the literature of the suitable optical data for the OAs is shown in Table 1. At present, suitable multicolor photometry is available only for 17 OAs. Moreover, the published photometry often comprises unorganized observations. This fact seriously complicates the reconstruction of the light curves in the various passbands and the analysis of the color variations over the whole event.

In order to determine meaningful color indices from the light curves of the OAs which often suffer from the shortcomings mentioned above, the light curves of the

\footnotetext{
1 Available at the URL:

http://gcn.gsfc.nasa.gov/gcn/gcn3_archive.html

2 http://www.aip.de/People/JGreiner/grbgen.html
} 

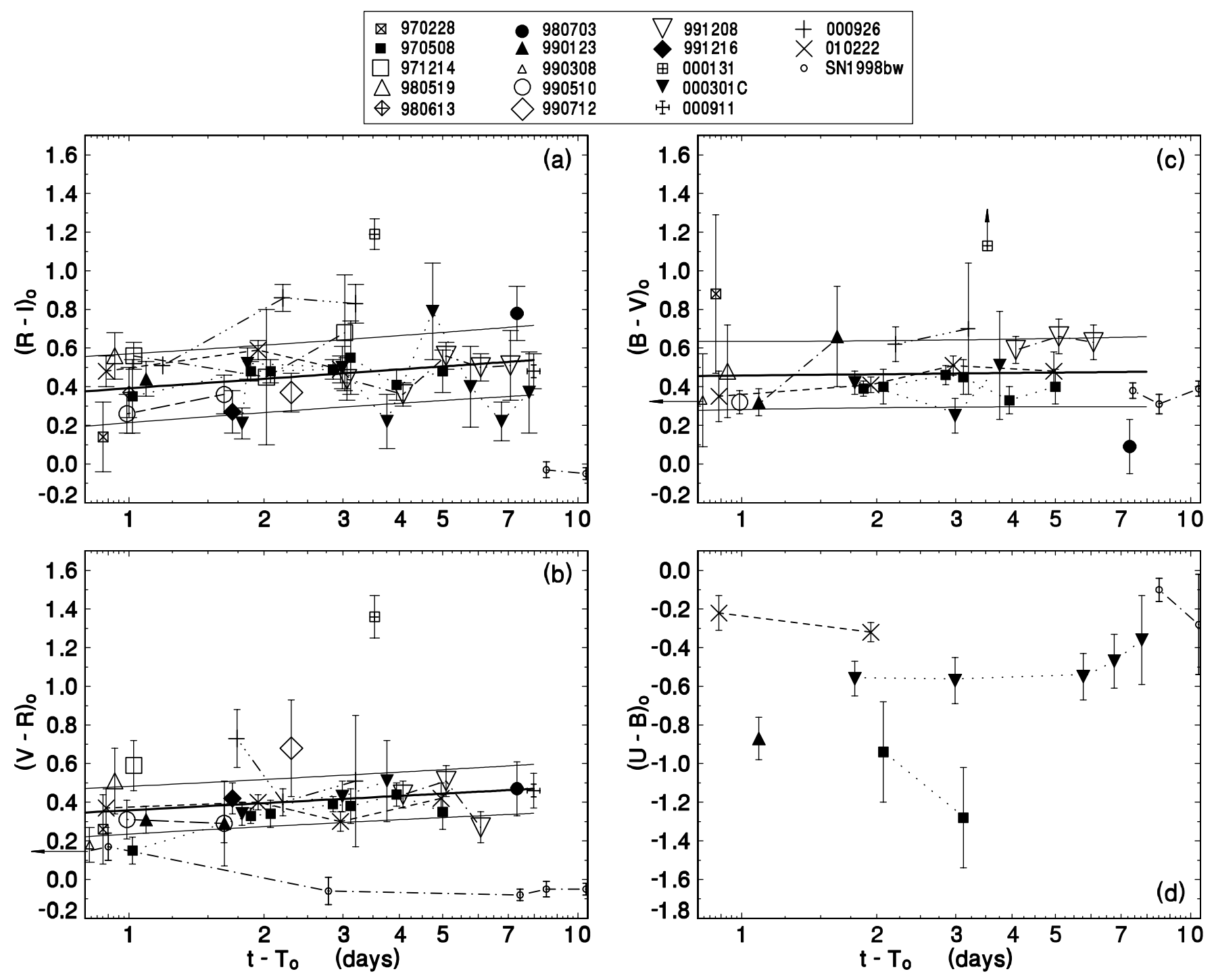

Fig. 1. Temporal evolution of the color indices of the respective afterglows. $t-T_{0}$ is the time interval in the observer frame elapsed from the corresponding GRB. Colors of all OAs correspond to their final decline branch with the exception of the first point of GRB970508. Only observations within $t-T_{0}<10$ days were plotted. The error bars denote the standard deviation. The color indices of a given OA are connected by lines for convenience. The fit to the whole ensemble of the OAs, except for the largely outlying GRB000131, is also shown (thick solid line) along with its standard deviation (thin solid line). The OA of GRB990308 $\left(t-T_{0}=0.14\right.$ days) falls out of scale in Figs. bc but is included in the fits. The colors of SN1998bw are shown just for comparison and are not included in the fits.

individual OAs in all available passbands were plotted in linear time scale and critically examined. It emerged that in most cases the light curves are free of complicated significant rapid changes on the time scale of hours to a few days. The mean course of the light curve could therefore be determined. If several measurements in a given filter were obtained within a few hours and had comparable standard deviations of brightness (quoted in the literature) then a centroid was calculated. If the standard deviations were largely discordant then just the most accurate measurements were selected for further analysis. It emerged that the color indices could be determined directly from the measurements (centroids) if the observations in the respective passbands were secured within about an hour in early phase of the OA $\left(t-T_{0}<3\right.$ days $)$ and even within a single night in later phases. In some cases an interpolation between the measurements, obtained in the neighbouring nights, was used, particularly when the coverage of the light curve in some passbands was denser than in the others. The standard deviation of each color index was calculated from the errors of the observations quoted in the literature. This procedure enabled us to obtain at least one color index for each of the 17 afterglows. Examination of the light curves of the OAs in the respective filters revealed that the available data allow reliable curves to be constructed in a given passband for $t-T_{0}<10$ days in most cases (except for the $R$-band which enabled to trace the evolution of the OAs to fainter brightness and 
Table 1. Summary of literature used for the construction of the light curves of the optical afterglows. Only those GRBs, for whose OAs at least one color index for $t-T_{0}<10$ days could be determined, are listed.

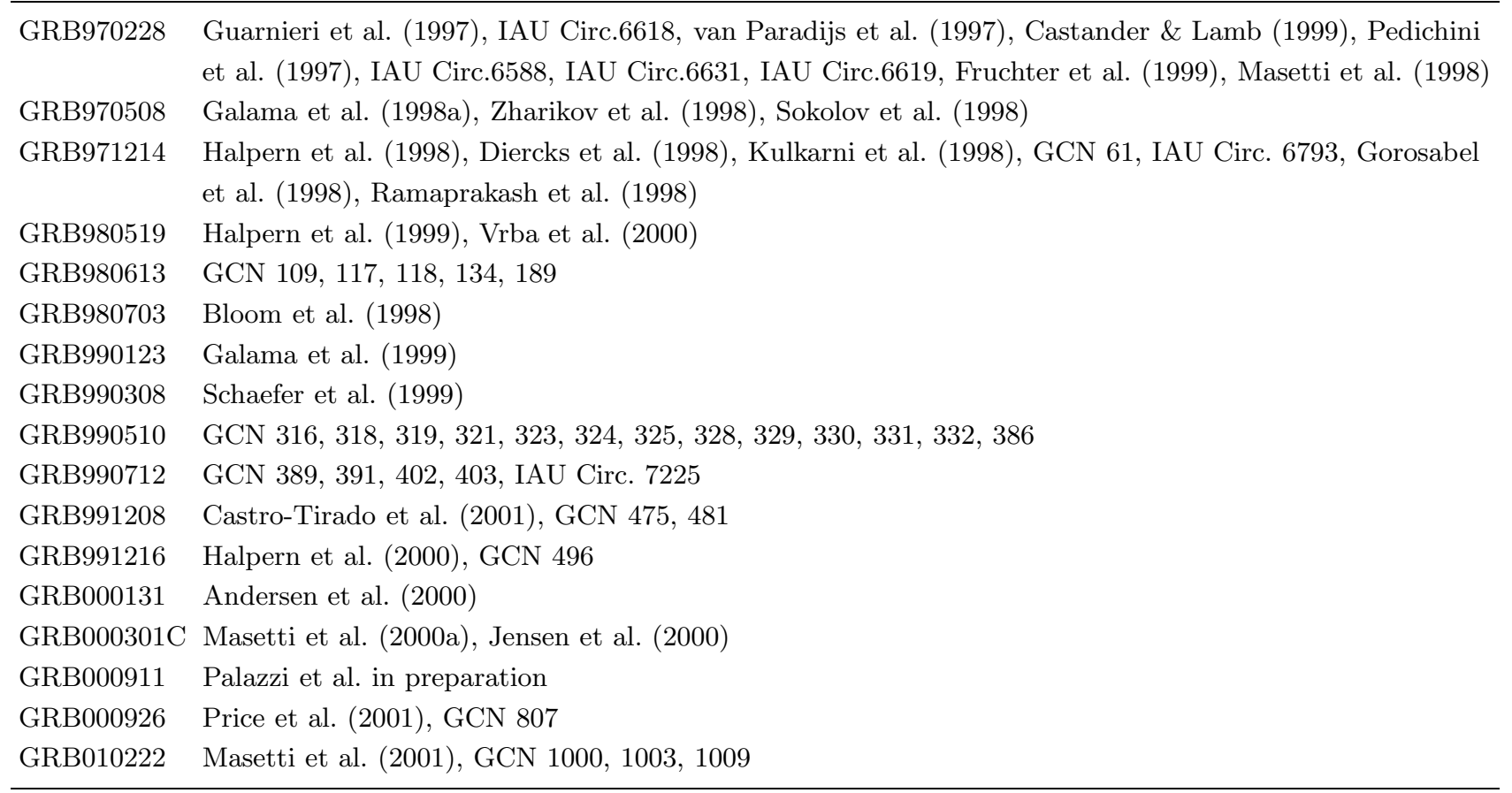

hence to longer $t-T_{0}$ ). We therefore decided to limit our analysis of the color indices to the points obtained within $t-T_{0}<10$ days. The color indices were corrected for the Galactic reddening according to the maps by Schlegel et al. (1998). These color indices of the OAs along with their standard deviations are listed in Table 2.

The color indices of the OAs were not corrected for the contribution of the host galaxies. This can be justified by the following reasons: we limit our analysis of the colors to the points obtained within $t-T_{0}<10$ days where the OAs were brighter than their hosts by several magnitudes, that means that the light contribution of the host galaxies is quite small and can be neglected.

Also, we did not take into account any redshift correction, which would be possible only if we had a correct a priori knowledge of the emerging spectrum of the OAs. Nevertheless, we will show that, despite the lack of a redshift correction, the colors of most OAs cluster around well determined values.

\subsection{The color diagrams of the afterglows}

Figures 1a,b,c,d show the available color indices $(R-I)_{0}$, $(V-R)_{0},(B-V)_{0}$ and $(U-B)_{0}$ of the 17 afterglows, plotted versus the time interval $t-T_{0}$ in the observer frame, elapsed since the corresponding GRB event at $T_{0}$. $T_{0}$ refers to the moment of the onset of the GRB. Only indices of the OAs within 10 days from the GRB trigger are plotted along with their standard deviations. $t-T_{0}$ is measured in the observer frame here in order to include also the color indices of those OAs for which no redshift $z$ is available. The colors of all OAs correspond to their typical power-law decline branch, with the exception of the first point of GRB970508. The color indices of the whole ensemble of OAs, displayed in a given panel, were also merged into a common file and fitted with a linear function to see if any evolution occurs in the first 10 days after the GRB. This fit is also shown in Figs. 1a,b,c as the thick solid line along with its standard deviation (thin solid lines). The data in Fig. 1d were not fitted because the available $(U-B)_{0}$ indices of the OAs are not numerous enough to enable meaningful fitting. Because the properties of SN1998bw, the possible counterpart of GRB980425, are markedly different from the remaining OAs, its colors are shown just for comparison and were not included in the fits. The colors of SN1998bw were determined from the light curves presented by Galama et al. (1998b). The color indices of all OAs, except GRB000131, occupy a narrow belt in Figs. 1a,b,c and the fits clearly demonstrate that the evolution of $(R-I)_{0},(V-R)_{0}$ and $(B-V)_{0}$ of these OAs is negligible over the considered time interval. The slight non-zero slope of the fits is of the order of 0.2 mag per 10 days and stays within their standard deviation. Although the color indices display some scatter, they mostly lie within the observational errors. We are therefore very cautious in drawing strong conclusions about the "fine structure" of the color evolution of the OAs from the available data. Instead, we will concentrate on analysis of the comprehensive properties of the whole ensemble of the color indices. The fact that the color indices of the OAs 
Table 2. The color indices of the optical afterglows of the GRBs, determined from the available data. Julian Date of the color index in the form JD-2400000 is given along with the time interval $t-T_{0}$ in the observer frame, elapsed from the corresponding GRB trigger. Only the color indices for $t-T_{0}<10$ days are listed. The standard deviation of a given color index is given in the line below. The color indices are corrected for the Galactic reddening. The last two columns bring the redshift $z$ and the value of the interstellar Galactic reddening $E_{B-V}$.

\begin{tabular}{|c|c|c|c|c|c|c|c|c|c|c|}
\hline GRB & JD & $t-T_{0}$ & $(U-B)_{0}$ & $(B-V)_{0}$ & $(V-R)_{0}$ & $(R-I)_{0}$ & $(I-J)_{0}$ & $(J-K)_{0}$ & $z$ & $E_{B-V}$ \\
\hline 970228 & 50508.5 & 0.876 & & $\begin{array}{c}0.88 \\
\pm 0.41\end{array}$ & $\begin{array}{c}0.26 \\
\pm 0.18\end{array}$ & $\begin{array}{c}0.14 \\
\pm 0.18\end{array}$ & & & 0.695 & 0.2167 \\
\hline \multirow[t]{7}{*}{970508} & 50578.42 & 1.018 & & & $\begin{array}{c}0.15 \\
\pm 0.07\end{array}$ & $\begin{array}{c}0.35 \\
\pm 0.19\end{array}$ & & & 0.835 & 0.0494 \\
\hline & 50579.27 & 1.868 & & $\begin{array}{c}0.39 \\
\pm 0.04\end{array}$ & $\begin{array}{c}0.33 \\
\pm 0.04\end{array}$ & $\begin{array}{c}0.48 \\
\pm 0.05\end{array}$ & & & & \\
\hline & 50579.47 & 2.068 & $\begin{array}{l}-0.94 \\
\pm 0.26\end{array}$ & $\begin{array}{c}0.40 \\
\pm 0.09\end{array}$ & $\begin{array}{c}0.34 \\
\pm 0.07\end{array}$ & $\begin{array}{c}0.48 \\
\pm 0.06\end{array}$ & & & & \\
\hline & 50580.25 & 2.848 & & $\begin{array}{c}0.46 \\
\pm 0.05\end{array}$ & $\begin{array}{c}0.39 \\
\pm 0.04\end{array}$ & $\begin{array}{c}0.49 \\
\pm 0.05\end{array}$ & & & & \\
\hline & 50580.52 & 3.118 & $\begin{array}{l}-1.28 \\
\pm 0.26\end{array}$ & $\begin{array}{c}0.45 \\
\pm 0.09\end{array}$ & $\begin{array}{c}0.38 \\
\pm 0.09\end{array}$ & $\begin{array}{c}0.55 \\
\pm 0.19\end{array}$ & & & & \\
\hline & 50581.35 & 3.948 & & $\begin{array}{c}0.33 \\
\pm 0.07\end{array}$ & $\begin{array}{c}0.44 \\
\pm 0.06\end{array}$ & $\begin{array}{c}0.41 \\
\pm 0.08\end{array}$ & & & & \\
\hline & 50582.40 & 4.998 & & $\begin{array}{c}0.40 \\
\pm 0.09\end{array}$ & $\begin{array}{c}0.35 \\
\pm 0.09\end{array}$ & $\begin{array}{c}0.48 \\
\pm 0.11\end{array}$ & & & & \\
\hline \multirow[t]{3}{*}{971214} & 50798.50 & 1.025 & & & $\begin{array}{c}0.59 \\
\pm 0.13\end{array}$ & $\begin{array}{c}0.56 \\
\pm 0.07\end{array}$ & $\begin{array}{c}0.95 \\
\pm 0.20\end{array}$ & $\begin{array}{c}1.45 \\
\pm 0.28\end{array}$ & 3.418 & 0.016 \\
\hline & 50799.50 & 2.025 & & & & $\begin{array}{c}0.45 \\
\pm 0.35\end{array}$ & & & & \\
\hline & 50800.50 & 3.025 & & & & $\begin{array}{c}0.68 \\
\pm 0.30\end{array}$ & & & & \\
\hline 980519 & 50953.93 & 0.93 & & $\begin{array}{c}0.48 \\
\pm 0.24 \\
\end{array}$ & $\begin{array}{c}0.51 \\
\pm 0.17 \\
\end{array}$ & $\begin{array}{c}0.56 \\
\pm 0.12 \\
\end{array}$ & & & & 0.2667 \\
\hline$\overline{980613}$ & 50978.70 & 1.000 & & & & $\begin{array}{c}0.37 \\
\pm 0.13\end{array}$ & & & 1.096 & 0.087 \\
\hline 980703 & 51005.0 & 7.315 & & $\begin{array}{c}0.09 \\
\pm 0.14\end{array}$ & $\begin{array}{c}0.47 \\
\pm 0.14\end{array}$ & $\begin{array}{c}0.78 \\
\pm 0.14\end{array}$ & & $\begin{array}{c}1.64 \\
\pm 0.18\end{array}$ & 0.966 & 0.058 \\
\hline 990123 & $\begin{array}{l}51203.00 \\
51203.54\end{array}$ & $\begin{array}{l}1.092 \\
1.632\end{array}$ & $\begin{array}{l}-0.87 \\
\pm 0.11\end{array}$ & $\begin{array}{c}0.32 \\
\pm 0.07 \\
0.66 \\
\pm 0.26\end{array}$ & $\begin{array}{c}0.31 \\
\pm 0.07 \\
0.29 \\
\pm 0.22\end{array}$ & $\begin{array}{c}0.44 \\
\pm 0.09\end{array}$ & & & 1.60 & 0.016 \\
\hline 990308 & 51245.85 & 0.140 & & $\begin{array}{c}0.33 \\
\pm 0.24 \\
\end{array}$ & $\begin{array}{c}0.18 \\
\pm 0.09 \\
\end{array}$ & & & & & 0.023 \\
\hline$\overline{990510}$ & $\begin{array}{l}51309.86 \\
51310.50\end{array}$ & $\begin{array}{l}0.990 \\
1.630\end{array}$ & & $\begin{array}{c}0.32 \\
\pm 0.06\end{array}$ & $\begin{array}{c}0.31 \\
\pm 0.10 \\
0.29 \\
\pm 0.10\end{array}$ & $\begin{array}{c}0.26 \\
\pm 0.10 \\
0.36 \\
\pm 0.10\end{array}$ & & & 1.619 & 0.227 \\
\hline$\overline{990712}$ & 51374.5 & 2.300 & & & $\begin{array}{c}0.68 \\
\pm 0.25\end{array}$ & $\begin{array}{c}0.37 \\
\pm 0.10\end{array}$ & & & 0.430 & 0.0327 \\
\hline \multirow[t]{5}{*}{$\overline{991208}$} & 51523.75 & 3.060 & & & & $\begin{array}{c}0.44 \\
\pm 0.11\end{array}$ & & & 0.707 & 0.016 \\
\hline & 51524.77 & 4.080 & & $\begin{array}{c}0.59 \\
\pm 0.07\end{array}$ & $\begin{array}{c}0.44 \\
\pm 0.07\end{array}$ & $\begin{array}{c}0.36 \\
\pm 0.06\end{array}$ & & & & \\
\hline & 51525.78 & 5.090 & & $\begin{array}{c}0.66 \\
\pm 0.09\end{array}$ & $\begin{array}{c}0.51 \\
\pm 0.08\end{array}$ & $\begin{array}{c}0.56 \\
\pm 0.07\end{array}$ & & & & \\
\hline & 51526.77 & 6.080 & & $\begin{array}{c}0.63 \\
\pm 0.09\end{array}$ & $\begin{array}{c}0.27 \\
\pm 0.08\end{array}$ & $\begin{array}{c}0.50 \\
\pm 0.07\end{array}$ & & & & \\
\hline & 51527.77 & 7.080 & & & & $\begin{array}{c}0.51 \\
\pm 0.18\end{array}$ & & & & \\
\hline \multirow[t]{2}{*}{991216} & 51529.72 & 0.55 & & & & & & $\begin{array}{c}1.46 \\
\pm 0.10\end{array}$ & 1.02 & 0.52 \\
\hline & 51529.90 & 0.73 & & & & & & $\begin{array}{c}1.50 \\
\pm 0.08\end{array}$ & & \\
\hline
\end{tabular}


Table 2. continued.

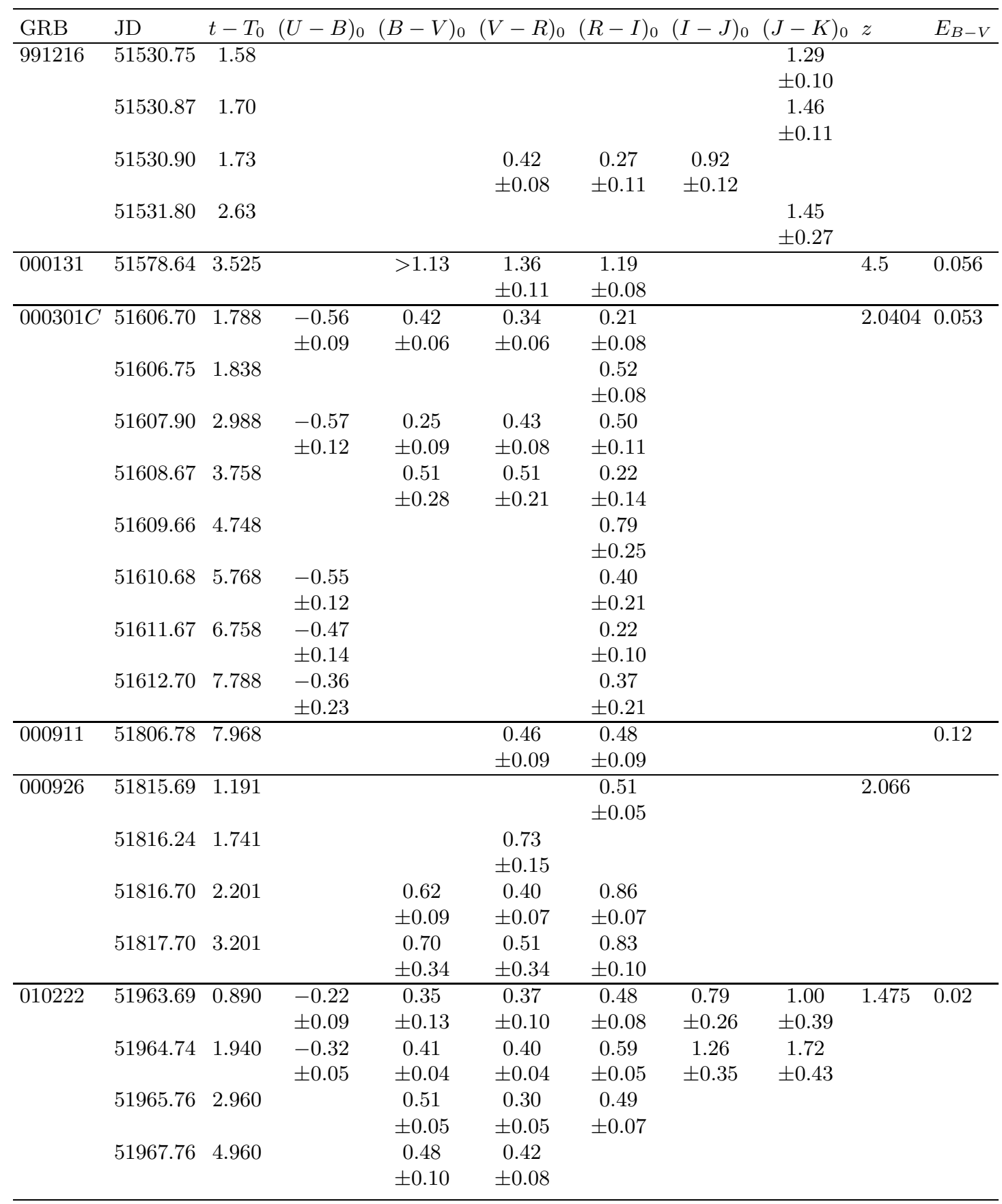

occupy a narrow belt in Figs. 1a,b,c suggests that common color-color diagrams can be built and that these diagrams are meaningful even if the observations of the various OAs come from different epochs after the GRB, albeit within 10 days from it.

Various color-color diagrams for the OAs, made with the data from Table 2, are shown in Figs. 2, 3 and 4. These diagrams have identical scales of the axes (and Figs. 2 and 3 also the zero points) to allow a direct comparison of the scatter among the plots. Usually both color indices which form a pair in the color-color diagrams come from measurements separated by at most one day. The mean colors (centroid) of the whole ensemble of OAs displayed in each diagram, including the standard deviations, are marked by crosses in Figs. 2 and 3. The error bars of the individual color indices, which are plotted in Figs. 1a,b,c,d, are not repeated in Figs. 2, 3 and 4 to avoid overcrowding of the plots.

Figures 2, 3 and 4 also show the representative reddening paths for $E_{B-V}=0.5$. The values of $z$ differ for the respective OAs such that observations in a given filter can therefore comprise radiation within a large range of 

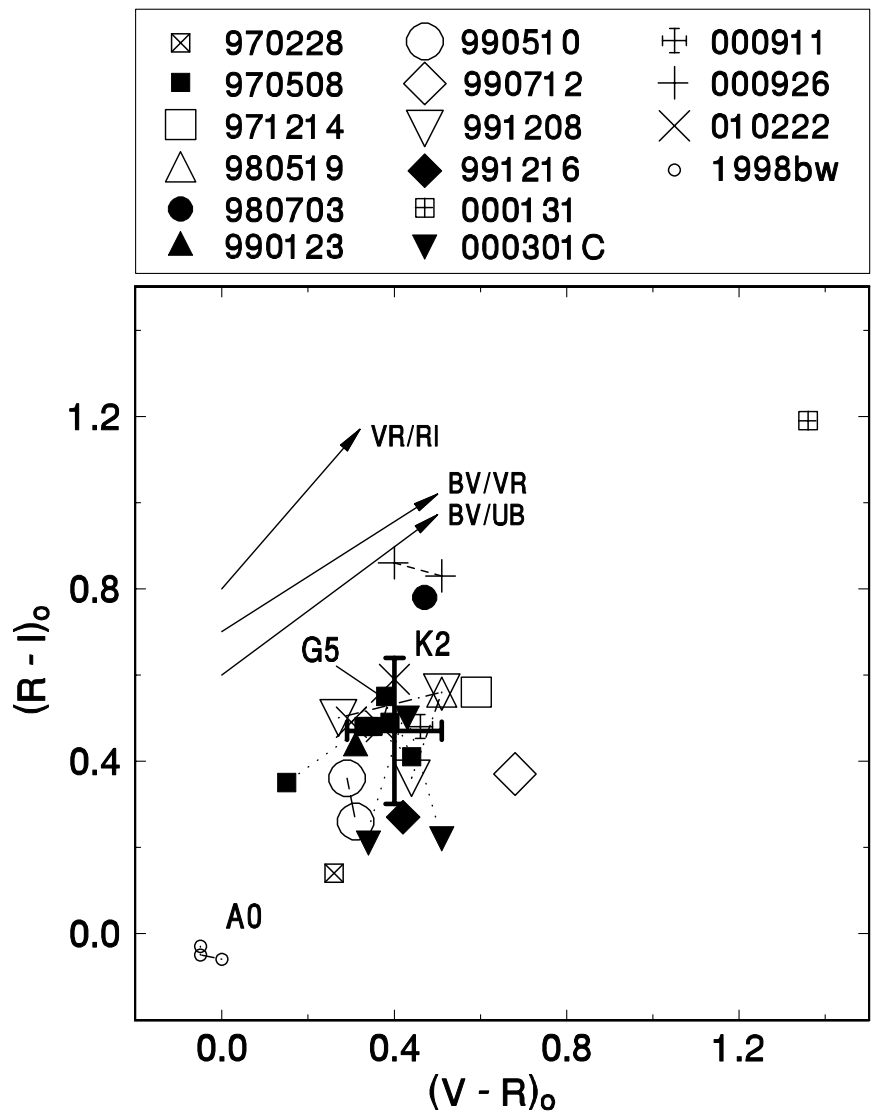

Fig. 2. $V-R$ vs. $R-I$ diagram of the afterglows. The color indices were corrected for the Galactic reddening. Only observations within $t-T_{0}<10$ days were plotted. Multiple indices of the same OA are connected by lines for convenience. The mean colors (centroid) of the whole ensemble of OAs (except for the largely outlying GRB000131), displayed in this diagram, including the standard deviations, are marked by the large cross. The colors of SN1998bw are shown just for comparison and were not included in calculation of the centroid. The representative reddening paths for $E_{B-V}=0.5$ are also shown. Positions of the main-sequence stars are included for comparison. See text for details.

wavelengths in the rest frame (see Sect. 2.1). Because the observer in a given passband will detect radiation at progressively shorter wavelengths with increasing redshift $z$, we decided to include the reddening paths, appropriate for $U-B, B-V, V-R$ and $R-I$, in all color-color diagrams. It can clearly be seen that both the lengths and directions of the vectors are similar for all reddening paths.

$V-R$ vs. $R-I$ diagram (Fig. 2): the color indices of all OAs, except for GRB000131, occupy a well localized region of the diagram and display no apparent scatter along the reddening path. Colors of some main-sequence stars which are also included can be used for the observational differentiation of the OAs from other objects.

$B-V$ vs. $V-R$ diagram (Fig. 3 ): all afterglows, except for GRB000131, occupy just a small region of the diagram again, without any scatter along the reddening path.

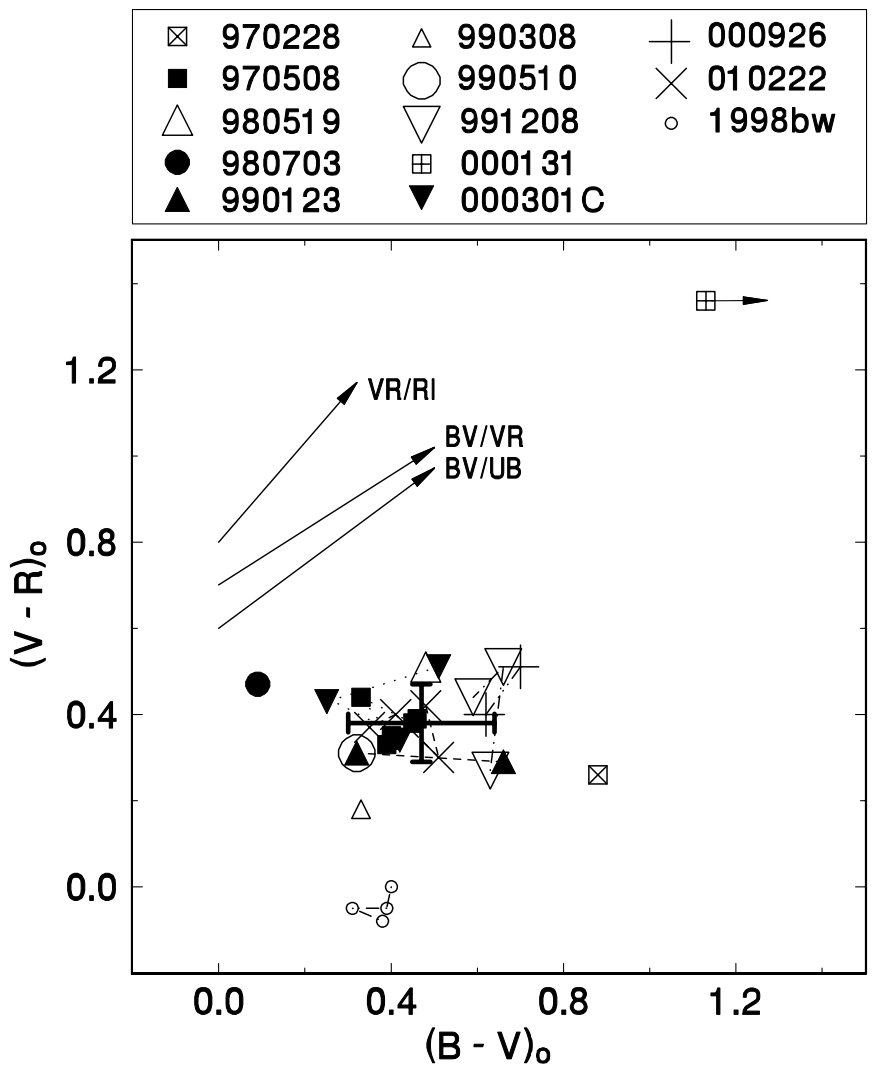

Fig. 3. $B-V$ vs. $V-R$ diagram of the afterglows. The arrangement is the same as in Fig. 2. The colors of GRB000131 and SN1998bw are shown just for comparison and were not included in calculation of the centroid.

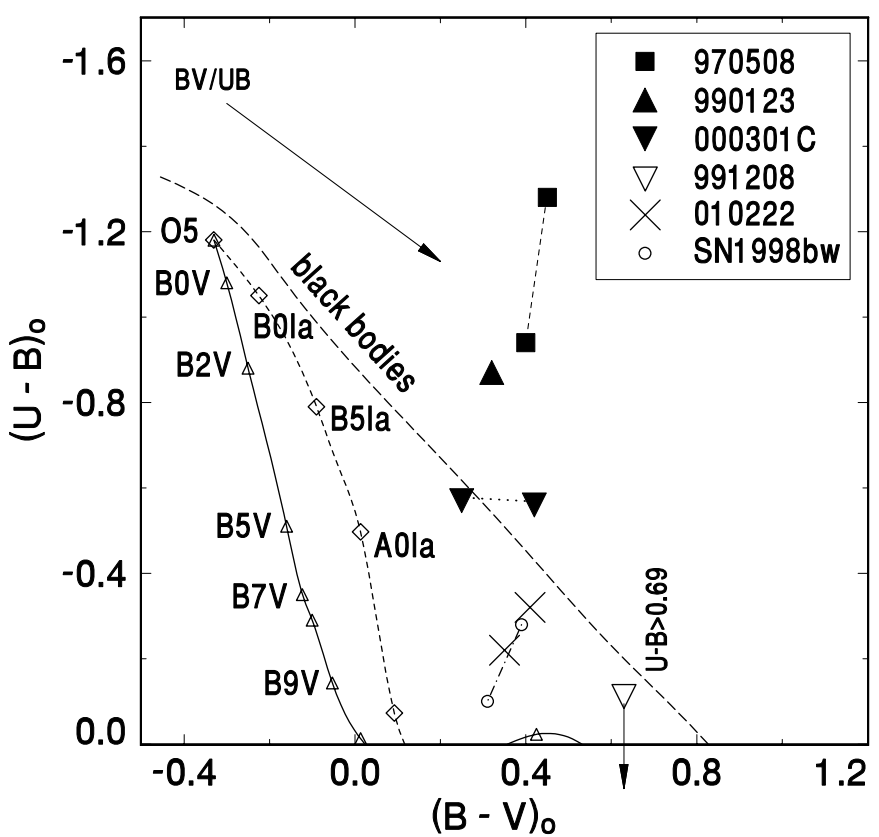

Fig. 4. $U-B$ vs. $B-V$ diagram of the afterglows. Positions of the main sequence stars, giants and the locus of black bodies are plotted - they can be used for the observational differentiation of OAs from other kinds of objects. The arrangement is similar to Fig. 2. See Sect. 2.1 for details. 

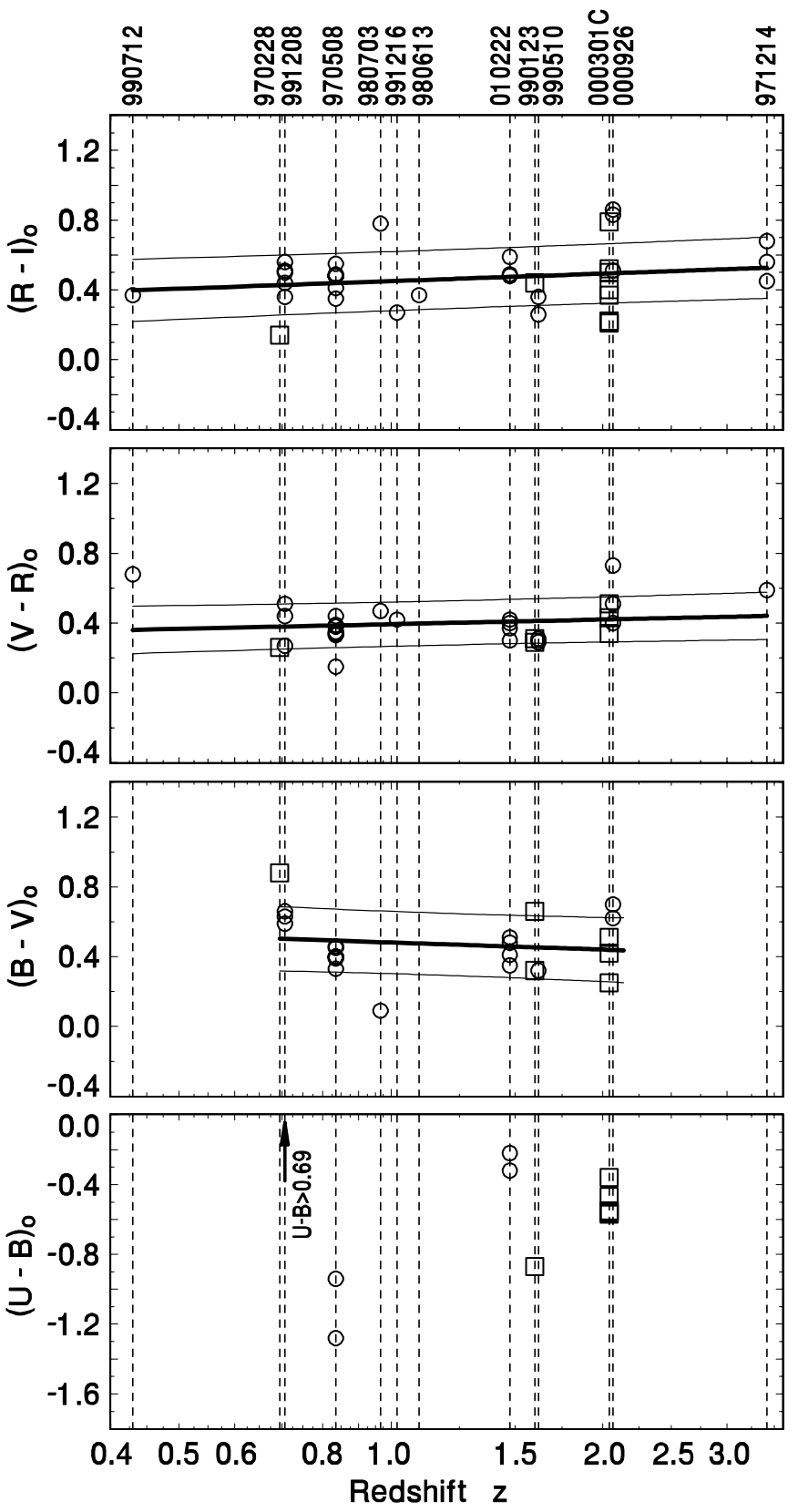

Fig. 5. The color indices of all OAs with known redshift $z$ plotted as a function of $z$. Only the OAs with $z<3.5$ are considered here. The fits and their $1 \sigma$ errors are marked by the thick and thin solid lines, respectively. The weight of each OA, used for each fit, was proportional to the number of available color indices. See Sect. 2.2 for details.

$U-B$ vs. $B-V$ diagram (Fig. 4): only four afterglows (GRB970508, GRB990123, GRB991208 and GRB000301C) allow determining the $U-B$ index or at least its limit. Notice the much larger scatter of the events in the $U-B$ direction than in $B-V$. Positions of the main sequence stars, giants and the locus of black bodies are plotted - they are useful for the observational differentiation of OAs from other objects.

$I-J$ and $J-K$ indices: they are available only for three and four OAs, respectively (Table 2). It can be seen from Table 2 that there is good agreement in $I-J$ and $J-K$ indices among all events for which these indices were measured.

Figures 2 and 3 then indicate a similar distribution for the optical colors of this OA sample $(B-V=0.47 \pm 0.17$, $V-R=0.40 \pm 0.13$ and $R-I=0.46 \pm 0.18)$. These average colors are consistent with a power-law shaped optical spectral distribution, in the form $F_{\nu} \propto \nu^{-\beta}$, with $\beta \sim 1$. This spectral shape naturally follows from the theoretical treatment of the GRB afterglow emission suggested by Sari et al. (1998) in the framework of the "fireball" model (see Sect. 3 for details).

\subsection{Dependence of the color index on the redshift $z$}

The observed passbands of OAs will be different from those in the rest frame because of the effects introduced by the redshift $z$. The values of $z$ of the OAs considered here lie in the range $0.43-4.5$, typical $z$ being around 1 . In the case of a complicated spectrum shape the color indices would be a function of $z$. The color indices of all OAs with known $z$ are plotted versus $z$ in Fig. 5. Only the OAs with $z<3.5$ are considered here; the case of GRB000131 $(z=4.5)$ is discussed in more detail in Sect. 3. The fits show that any dependence of the color on $z$ is weak and within the $1 \sigma$ errors for $(R-I)_{0},(V-R)_{0}$ and $(B-V)_{0}$. The only exception may be $(U-B)_{0}$.

The relation between the wavelength observed in a given passband and the rest-frame wavelength, radiated by the OA with known redshift $z$ can be seen in Fig. 6 . This diagram shows that the $(R-I)_{0},(V-R)_{0},(B-V)_{0}$ and $(U-B)_{0}$ indices represent the radiation between about $800 \AA$ and $5600 \AA$ in the rest frame.

The relation in Fig. 6, when compared with the strong clustering of the color indices in Figs. 2 and 3, clearly shows that the $(R-I)_{0},(V-R)_{0}$ and $(B-V)_{0}$ indices represent the radiation between about 1500 and $5500 \AA$ in the rest frame. On the other hand, the large scatter, observed in $(U-B)_{0}$, originates below $1500 \AA$ in the rest frame.

\subsection{Absolute brightness of the OAs}

Absolute brightness in the $R$-band $\left(M_{R_{0}}\right)$ for each OA was calculated in two steps: first, by determining the luminosity distance $D_{\mathrm{L}}$ from the redshift $z$, using Eq. (15.3.25) of Weinberg (1972) and $H_{0}=60 \mathrm{~km} \mathrm{~s}^{-1} \mathrm{Mpc}^{-1}$; then, by applying the distance modulus formula using $D_{\mathrm{L}}$ and the observed $R$-band magnitude corrected for the Galactic reddening according to the maps by Schlegel et al. (1998). The time intervals $t-T_{0}$ in the observer frame were transformed into the rest frame times $\left(t-T_{0}\right)_{\text {rest }}$. The resultant light curves for OAs with known $z$ are displayed in Fig. 7a. Here the brightness of OAs in Fig. 7a was also corrected for the light contribution of the host galaxy because these $R$-band light curves extend far behind $t-T_{0}<10$ days, used as a limit for the color indices, and the brightness 


\begin{tabular}{|c|c|c|c|}
\hline $\begin{array}{l}\lambda_{I}=8090 \mathrm{~A} \\
\lambda_{R}=6602 \mathrm{~A} \\
\lambda_{V}=5500 \mathrm{~A} \\
\lambda_{B}=4393 \mathrm{~A} \\
\lambda_{U}=3372 \mathrm{~A}\end{array}$ & $\begin{array}{l}1 \ldots 970228 \\
2 \ldots . .970508 \\
3 \ldots . .971214 \\
4 \ldots 980613 \\
5 \ldots 980703\end{array}$ & $\begin{array}{l}6 . . .990123 \\
7 \ldots . .990510 \\
8 \ldots . .990712 \\
9 \ldots . .991208 \\
10 . .991216\end{array}$ & $\begin{array}{l}11 \ldots 000131 \\
12 . . .000301 C \\
13 . .000926 \\
14 \ldots 010222\end{array}$ \\
\hline
\end{tabular}

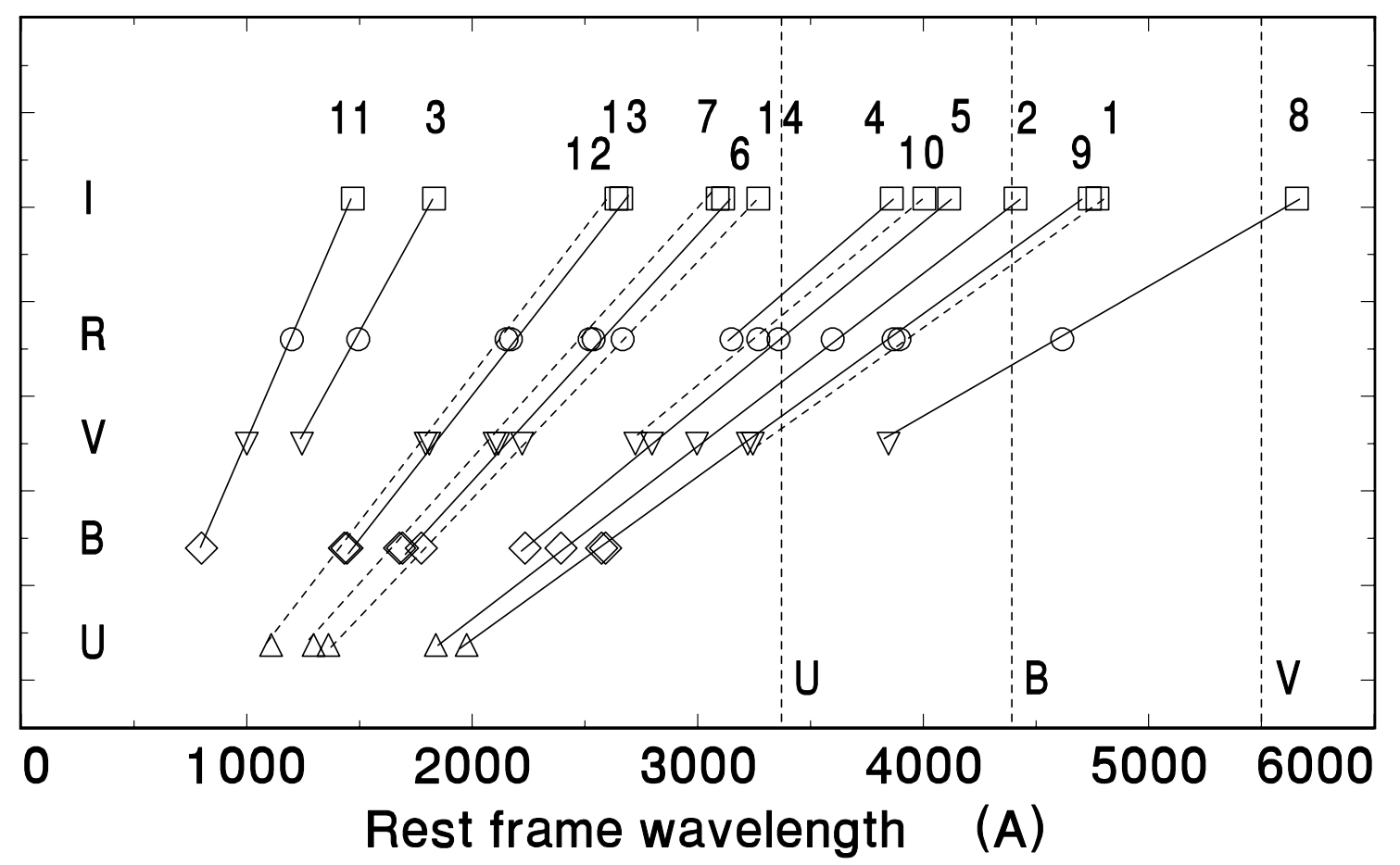

Fig. 6. Relation between the wavelength of radiation observed in a given passband and the rest-frame wavelength, radiated by the OA with known redshift $z(U$-filter - triangles; $B$-filter - diamonds; $V$-filter - inverted triangles; $R$-filter - circles; $I$-filter squares). Only those OAs for which at least one observation in a given passband exists are included in the plot. See Sect. 2.2 for details.

of the OA decreased so much that the light of the host could not be neglected. We note, however, that in most cases the host galaxy noticeably affects the brightness of OA only in its very late phase $\left(t-T_{0}\right.$ of the order of tens of days).

It is desirable to examine whether the spread of the absolute brightnesses $M_{R_{0}}$ of the respective OAs in Fig. 7a is real or if it is caused mainly by the shifts of the effective wavelength $\lambda$ due to the different redshifts $z$. The observer in the $R$ band will detect radiation originated at progressively shorter wavelengths in the GRB rest frame with increasing redshift $z$. As it will be argued below, the spectra of the OAs are very smooth and very similar each to other. Because generally the intensity may be somewhat different at various $\lambda$ due to the slope of the spectrum, the shift of the effective $\lambda$ will introduce some change of brightness. $K$-correction is possible only if the shape of the spectrum is known a priori. First, we decided to make a model-independent test which does not impose any assumptions on the shape and the slope of the spectrum of OAs. A correlation between the redshift $z$ and $M_{R_{0}}$ (measured for some $\left(t-T_{0}\right)_{\text {rest }}$, identical for all OAs) is expected if the spread of brightness were caused purely by the shift of $\lambda . M_{R_{0}}$ was measured at the same $\left(t-T_{0}\right)_{\text {rest }}=1.5$ days. All OAs, plotted in Fig. 7a, already achieved the decay branch of their light curve. The light curves of the decays of all OAs in Fig. 7a were fitted by the straight line because only the mean slope, averaged over the "fine" structure seen in some events, was investigated. $M_{R_{0}}$ was then read out from the fit. An extrapolation of the decay had to be used for the OA of GRB971214. The result is shown in Fig. $7 \mathrm{~b}$. It can readily be seen that there is no apparent correlation between $z$ and $M_{R_{0}}$.

A further correction of the $M_{R_{0}}$ of the OAs in this sample has been performed by assuming that the OA spectra can be modeled using a power law with index $\beta \sim 1$, as suggested by the results reported in Sect. 2.1, and keeping into account which rest-frame band is corresponding to the $R$ band as seen in the observer frame. In particular, as the observer frame $R$ bands of the OAs in this sample mainly lie within the $B$ optical band and around $2000 \AA$ in the UV region (e.g. the $U V W 2$ filter 
of the Optical Monitor onboard the XMM-Newton satellite; Dahlem 2000), we first computed the color indices $U V W 2-R, U-R, B-R$ assuming $\beta \sim 1$. In this hypothesis, they are $\sim-0.3$ (the rest-frame wavelength of the OAs $\left.\lambda_{\text {rest }}=1400-2800 \AA\right), \sim 0.2\left(\lambda_{\text {rest }}=2800-3750 \AA\right)$ and $\sim 0.9\left(\lambda_{\text {rest }}=3750-4800 \AA\right)$, respectively. Next, we corrected the observed absolute $R$ magnitude by subtracting the appropriate color index value from $M_{R_{0}}$ as determined by using the luminosity distance and the distance modulus formulae. This is some sort of zeroth order $k$-correction introduced to make an attempt at taking into account the shift on the OA optical spectra produced by the cosmological redshift. In this way we can better compare the "real" $R$-band absolute magnitudes of this set of OAs (Fig. 7c).

\section{Discussion}

We have shown that the color indices of most OAs occupy the well defined belts, when plotted as a function of time, especially for $t-T_{0}<10$ days, and that the time evolution of these indices is negligible during this interval. Most afterglows appear to concentrate in the well localized regions of $V-R$ vs. $R-I$ and $B-V$ vs. $V-R$ diagrams. Colors of all OAs analyzed here correspond to their final decline branch with the exception of the first point of GRB970508. The color indices $(R-I)_{0}=0.46 \pm 0.18$, $(V-R)_{0}=0.40 \pm 0.13,(B-V)_{0}=0.47 \pm 0.17$ appear to be typical. OAs therefore appear quite red in the spectral region between the $I$ and $B$-filter (colors similar to mid-G or early $\mathrm{K}$ stars). On the other hand, the $(U-B)_{0}$ index of the OAs is negative with a large scatter and the position of the afterglows in $U-B$ vs. $B-V$ diagram is often quite different from other sources. These specific colors can be used to distinguish genuine OAs related to GRBs from another types of objects in optical GRB searches.

The results reported here are thus extremely significant from the point of view of optical searches for $\gamma$ ray bursters. First, the well defined color of OAs allows, together with the power law decline, to distinguish the real OAs of GRBs from the other types of astrophysical objects. So far, only the rapid fading behavior has served to distinguish the genuine afterglows. So we have another tool now, enabling one to analyze images taken in various optical passbands quickly for possible optical counterparts of GRBs, without waiting for the following night to confirm the rapid object fading. Secondly, the well focused position of OAs in color-color diagrams represents a very important tool to consider independent (on satellite projects) optical searches for OAs of GRBs. Although there are suitable databases available for this kind of analyses such as the deep UKSTU archival plates, the search for real OAs was difficult because of high background level of other types of variable phenomena not related to GRBs. The combination of fading profile with the color information may be used as a very powerful tool to search for real OAs in suitable databases with color information such as the UKSTU archive. It should be noted that the actual rate of OAs may exceeds those of GRBs due to
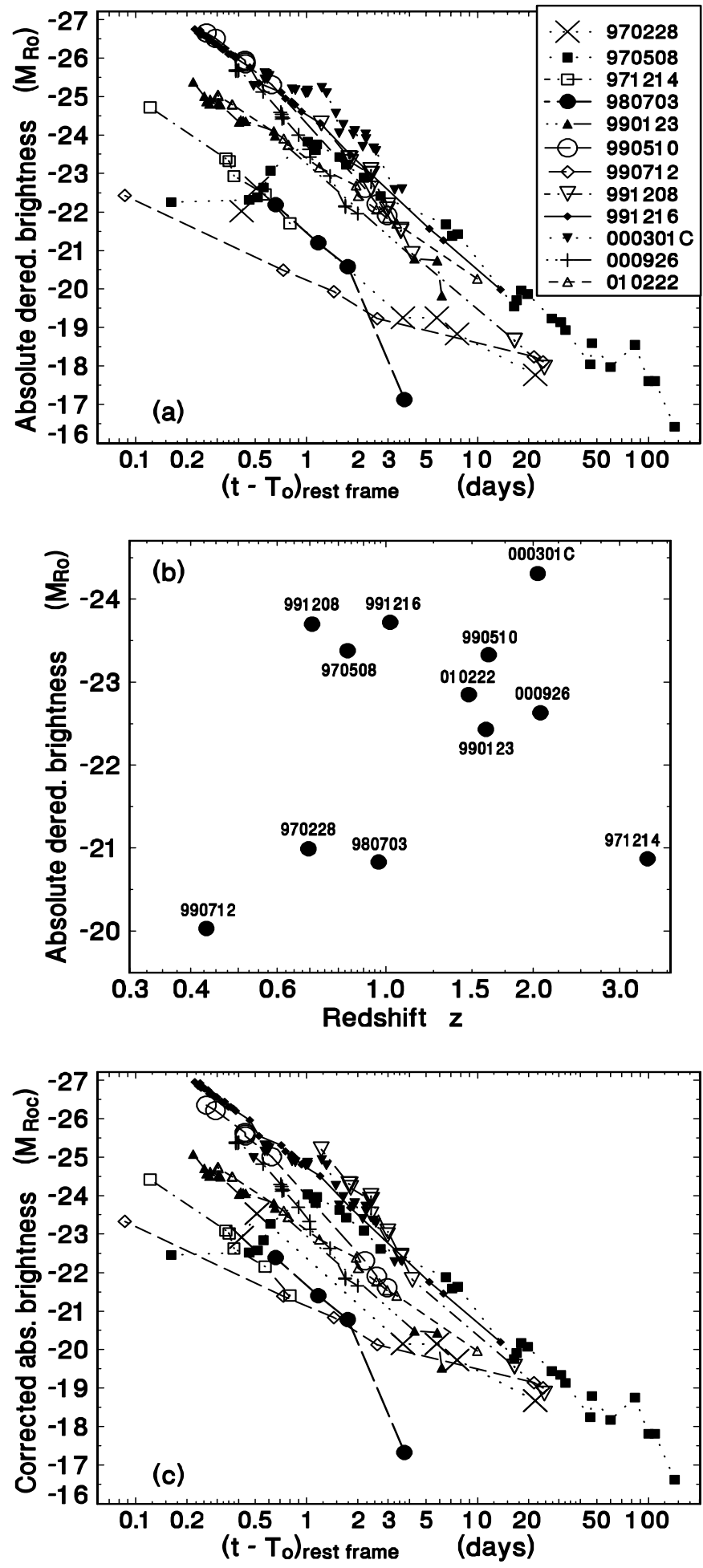

Fig. 7. a) Absolute brightness of the OAs in the observed $R$-band, corrected for the Galactic reddening and the light contribution of the host galaxy. The time intervals $t-T_{0}$ in the observer frame were transformed into the rest frame $\left(t-T_{0}\right)_{\text {rest }}$. b) Absolute brightness $M_{R_{0}}$ of the OAs, measured at $\left(t-T_{0}\right)_{\text {rest }}=1.5$ days, plotted versus redshift $z$. A correlation between $z$ and $M_{R_{0}}$ would be expected if the spread of brightness of the respective light curves in Fig. 7a were caused purely by the shift of $\lambda$. c) The same as in Fig. 7a but zeroth order $k$-correction was applied to the observed $R$ magnitudes of the respective OAs. See Sect. 2.3 for details. 
different beaming in $\gamma$-rays and in optical (Hudec 2000). Moreover, the OAs detected by optical searches may provide very precise localizations of GRBs, and hence allow detailed studies of their host galaxies. On the other hand, the OAs rates and/or limits provided by these analyses could provide constraints on the time and/or wavelength dependent beaming in GRBs, and hence contribute to the understanding of the physical model of the events.

The results presented here also confirm the importance of color information in the strategy of the optical afterglow searches and analyses. Not only the fading profile, but also the color information may yield a valuable physical conclusion regarding the model, the origin, and the position of GRBs. We hence stress that it is important to provide further optical observations of OAs in a well organized way. If possible, the observations should be carried out with standard filters, and various passbands should be taken immediately i.e. during the same night.

The fact that most OAs concentrate in the well defined regions of the color-color diagrams with the standard deviations just about $0.13-0.18 \mathrm{mag}$ (except for $\left.(U-B)_{0}\right)$, despite the smearing introduced by the different redshifts $z$ and the corresponding shifts of the passbands, implies that the spectral shape of OAs is very smooth, with no bumps or strong lines, within the observed $I$ to $B$ passbands. The relation between the wavelength of radiation observed in a given passband and the rest-frame wavelength, radiated by the OA (Fig. 6), shows that the $(R-I)_{0},(V-R)_{0}$, $(B-V)_{0}$ indices which display very small scatter, represent the radiation within about 2000 and $5600 \AA$ in the rest frame. Also the slope of the spectrum can be supposed to be almost constant and may have a powerlaw shape $F_{\nu} \propto \nu^{-\beta}$ in this spectral region. If $\beta$ is similar for practically all OAs, then the redshift effect on the ratio between luminosities in different wavebands (i.e. color indices) is very small, as observed. Indeed, the average colors in Figs. 2 and 3 are consistent with a power-law shaped optical spectral distribution with $\beta \sim 1$. This spectral shape naturally follows from the theoretical treatment of the GRB afterglow emission suggested by Sari et al. (1998) in the framework of the "fireball" model. In this model the luminosity comes from a relativistic expanding and decelerating shell which radiates via synchrotron emission. The value found for $\beta$ is fully compatible, within this model, with an electron power-law energy distribution with index $p \sim 2.5$, as observationally found by e.g. Frontera et al. (2000) from the spectral analysis of GRB prompt highenergy emission.

The available color indices of the OAs represent relatively late stage of the event because they come from $t-T_{0} \geq 0.14$ days. They are thus expected to represent the blast wave already moving rather spherically, with just a little beaming (Piran 1999). In the framework of the fireball model, these color indices represent the processes in which only the forward external shocks play a role. The similarity of the color indices of most OAs considered here suggests that the properties of these shocks remain quite similar for the respective events.

It is remarkable that the color indices $(R-I)_{0}$, $(V-R)_{0}$ and $(B-V)_{0}$ do not evolve within $t-T_{0}<$ 10 days (Fig. 1) although the brightness of all OAs declines by several magnitudes during this time interval (Figs. 7a,c). It implies that the shape of the spectrum does not change significantly while the luminosity of the OAs decreases by a large amount.

The absence of correlation between $z$ and $M_{R_{0}}$ together with the very small scatter of the color indices of the respective OAs allows one to conclude that the smearing of $M_{R_{0}}$, introduced by the different values of $z$, does not significantly alter the scatter of $M_{R_{0}}$ of the various events. The range of $M_{R_{0}}$ of the OAs in Fig. $7 \mathrm{c}$ for $\left(t-T_{0}\right)_{\text {rest }}=0.25$ days where most OAs already reached their final decline branch is about $4.3 \mathrm{mag}$, from $M_{R_{0}}=-26.5$ to -22.2 . It implies the range of luminosities about 1:50.

The fact that the spectra are similar although the luminosity of OAs appears to be different is most likely due to the total initial energy of the afterglow. As a matter of fact, the spectral shape of the fireball model, as modeled by Sari et al. (1998), does not depend on the input energy while the luminosity of the afterglow at a particular epoch does depend on it. So, the higher the GRB input energy is, the brighter the OA is; this would also suggest one more time that GRBs and their afterglows are not standard candles.

In any case, although it is apparent also from Figs. 7a,c that the OAs are not standard candles, it is interesting to note that five OAs have very similar $M_{R_{0}}$ for $1<\left(t-T_{0}\right)_{\text {rest }}<3$ days (GRB970508, 990123, 990510, 991208, 000301C).

The general slope of the decay branches of OAs is largely independent of $M_{R_{0}}$ and the light curves of all OAs considered here are almost parallel. This means that the difference in the luminosities of the respective OAs persists through the decline, at least for $0.2<\left(t-T_{0}\right)_{\text {rest }}<$ 20 days. The OA of GRB990712 might have a slower decay than the others but its host galaxy was exceptionally bright - the brightness of an OA is then largely dependent on the exact value of the brightness of the host. The different observed mean decay slopes therefore can be attributed mostly to the relativistic effects - most these differences disappear when transformation to the rest frame is made.

The strong concentration of the color indices in the color-color diagrams (Figs. 2 and 3) suggests that the intrinsic reddening (i.e. in their host galaxies) must be quite similar for all OAs and, moreover, that this reddening is likely to be rather small. The reason is that in the case of a large reddening it would be quite unlikely to obtain such similar values of absorption in all cases. Notice that there is no apparent scatter of the color indices of the OAs (except for GRB000131) along the reddening path, depicted in Figs. 2 and 3. The scatter in the 
$U-B$ vs. $B-V$ diagram (Fig. 4 ) is large but only in the $U-B$ direction which is inconsistent with the interstellar reddening. All these lines of evidence therefore imply that most GRBs whose afterglows are analyzed here are unlikely to come directly from the inner (densest) parts of the star-forming regions. However, that does not exclude the possibility that these GRBs originate on "our side" of a structured star-forming region. Alternatively, the density and the dust abundance of the local interstellar medium might be substantially reduced by the intense high-energy radiation of the GRB trigger, as modeled by Waxman \& Draine (2000).

We are however aware that in some cases the OAs appear to have steeper optical spectra (and thus redder colors), as, for example, GRB980329 (Palazzi et al. 1998), GRB990705 (Masetti et al. 2000b), GRB000131 (Andersen et al. 2000), GRB000418 (Klose et al. 2000), GRB000630 (Fynbo et al. 2000). These GRBs show $\beta \sim 2$, or higher. This is most likely due to strong local absorption in the burst environment (as in the cases of GRB990705 and GRB000418) or very high redshift (as for GRB000131), or possibly both (the case of GRB980329; Fruchter 1999). Thus, our results suggest that the sample of OAs considered here is very little affected by reddening effects induced by both strong local absorption or high redshift, i.e. here we are dealing with GRB afterglows which are at $z<4$ and not deep inside dense dust clouds in their host galaxies. At present, the available data enable the determination of the color indices of the redder OAs according to the criteria from Sect. 2 only for the OA of GRB000131. Nevertheless, the strong concentration of the color indices of the OAs in Figs. 2 and 3 allows one to infer that there may not be smooth transition between the events considered in our analysis and these OAs with steep optical spectra.

The supernova SN1998bw, which is a possible optical counterpart of GRB980425, is markedly different from the remaining OAs in several respects. While the OAs from Table 1 lie at the cosmological distances $(z \geq 0.43)$, the much smaller redshift of SN1998bw $(z=0.0085)$ suggests a considerably smaller distance and hence much lower absolute brightness. Also the light curve of SN1998bw largely differs from the remaining OAs because the brightness of SN1998bw was still steadily increasing within $t-T_{0}<10$ days while all other OAs already achieved their decline branch and faded by several magnitudes. Generally, the color indices of SN1998bw in Figs. 1, 2, 3 and 4 suggest the shape of spectrum which is different from the other OAs and cannot be explained purely by the different values of $z$.

Comparison of the colors of OAs and their evolution with the behaviour of supernovae in general may be fruitful because both cases presumably represent explosive processes. At this stage, comparison of the general trends in the color evolution of the kinds of objects seems to be more suitable than a detailed comparison of what happens on the absolute time scale. Let us confine to $t-T_{0}<10$ days for OAs (brightness of SNe typically falls by about the same amount after more than 100 days). The small spread of colors of OAs is a feature similar to the type Ia SNe where after a rapid rise (up to approx. 30 days after $T_{\max }$ ) $B-V$ of the individual SNIa agrees within about $0.2 \mathrm{mag}$ and slowly decreases linearly with time during a large part of the decline $\left(30<t-T_{\max }<90\right.$ days) (Phillips et al. 1999). On the contrary, SNII display a large spread in $B-V$ and this index generally increases by almost 1 mag during the first 100 days (Patat et al. 1994).

Acknowledgements. This research has made use of NASA's Astrophysics Data System Abstract Service. We acknowledge the support by the project KONTAKT ME 137 and ES02 by the Ministry of Education and Youth of the Czech Republic and the grant 205/99/0145 of the Grant Agency of the Czech Republic. We also acknowledge the CNR-AVČR Joint Research Program No. 3 (1998/2000).

\section{References}

Andersen, M., Hjorth, J., Pedersen, H., et al. 2000, A\&A, 364, L54

Bloom, J. S., Frail, D. A., Kulkarni, S. R., et al. 1998, ApJ, 508, L21

Castander, F. J., \& Lamb, D. Q. 1999, ApJ, 523, 593

Castro-Tirado, A. J., Sokolov, V. V., Gorosabel, J., et al. 2001, A\&A, 370, 398

Dahlem, M. 2000, XMM User's Handbook, version 1.2, available at http://xmm.vilspa.esa.es/user/uhb/XMM_UHB

Diercks, A. H., Deutsch, E. W., Castander, F. J., et al. 1998, ApJ, 503, L105

Frontera, F., Amati, L., Costa, E., et al. 2000, ApJS, 127, 59

Fruchter, A. S. 1999, ApJ, 512, L1

Fruchter, A. S., Pian, E., Thorsett, S. E., et al. 1999, ApJ, 516, 683

Fynbo, J. U., Jensen, B. L., Gorosabel, J., et al. 2000, A\&A, 369,373

Gorosabel, J., Castro-Tirado, A. J., Willott, C. J., et al. 1998, A\&A, 335, L5

Galama, T. J., Groot, P. J., van Paradijs, J., et al. 1998a, ApJ, 497, L13

Galama, T. J., Vreeswijk, P. M., van Paradijs, J., et al. 1998b, Nature, 395, 670

Galama, T. J., Briggs, M. S., Wijers, R. A. M. J., et al. 1999, Nature, 398, 394

Guarnieri, A., Bartolini, C., Masetti, N., et al. 1997, A\&A, 328, L13

Halpern, J. P., Thorstensen, J. R., Helfand, D. J., \& Costa, E. 1998, Nature, 393, 41

Halpern, J. P., Kemp, J., Piran, T., \& Bershady, M. A. 1999, ApJ, 517, L105

Halpern, J. P., Uglesich, R., Mirabal, N., et al. 2000, ApJ, 543, 697

Hudec, R. 2000, in Gamma-Ray Bursts - 5th Huntsville Symposium, ed. R. M. Kippen, R. S. Mallozzi, \& G. J. Fishman, AIP Conf. Proc., 526

Jensen, B. L., Fynbo, J. U., Gorosabel, J., et al. 2000, A\&A, 370, 909

Klose, S., Stecklum, B., Masetti, N., et al. 2000, ApJ, 545, 271

Kulkarni, S. R., Djorgoski, S. G., Ramaprakash, A. N., et al. 1998, Nature, 393, 35 
Masetti, N., Bartolini, C., Guarnieri, A., \& Piccioni, A. 1998, in The Active X-ray Sky: Results from BeppoSAX and Rossi-XTE, ed. L. Scarsi, H. Bradt, P. Giommi, \& F. Fiore, Nuc. Phys. B Proc. Suppl. 69/1-3, 674

Masetti, N., Bartolini, C., Bernabei, S., et al. 2000a, A\&A, 359, L23

Masetti, N., Palazzi, E., Pian, E., et al. 2000b, A\&A, 354, 473

Masetti, N., Palazzi, E., Pian, E., et al. 2001, in press [astro-ph/0103296]

Palazzi, E., Pian, E., Masetti, N., et al. 1998, A\&A, 336, L95

Patat, F., Barbon, R., Cappellaro, E., \& Turatto, M. 1994, A\&A, 282, 731

Pedichini, F., Di Paola, A., Stella, L., et al. 1997, A\&A, 327, L36

Phillips, M. M., Lira, P., Suntzeff, N. B., et al. 1999, AJ, 118, 1766

Piran, T. 1999, Phys. Rep., 314, 575

Price, P. A., Harrison, F. A., \& Galama, T. J. 2001, ApJ, 549, L7

Ramaprakash, A. N., Kulkarni, S. R., Frail, D. A., et al. 1998, Nature, 393, 43

Rhoads, J. E. 2001, ApJ, 557, 943

Sari, R., Piran, T., \& Narayan, R. 1998, ApJ, 497, L17

Schaefer, B. E., Snyder, J. A., \& Hernandez, J. 1999, ApJ, 524, L103
Schlegel, D. J., Finkbeiner, D. P., \& Davis, M. 1998, ApJ, 500, 525

Šimon, V., Pizzichini, G., \& Hudec, R. 2000a, in Gamma-Ray Bursts - 5th Huntsville Symposium, ed. R. M. Kippen, R. S. Mallozzi, \& G. J. Fishman, AIP Conf. Proc., 526

Šimon, V., Hudec, R., Masetti, N., \& Pizzichini, G. 2000b, The Procedings of the 4th INTEGRAL Workshop, 4-8 September (Alicante, Spain), accepted

Šimon, V., Hudec, R., Masetti, N., \& Pizzichini, G., 2000c, in ed. F. Frontera, E. Costa, \& J. Hjorth, Gamma-Ray Bursts in the Afterglow Era: 2nd Workshop, A Joint CNR/ESO Meeting, C.N.R. Headquarters, Rome, Italy (October 17-20, 2000)

Sokolov, V. V., Kopylov, A. I., Zharikov, S. V., et al. 1998, A\&A, 334, 117

van Paradijs, J., Groot, P. J., Galama, T. J., et al. 1997, Nature, 386, 686

Vrba, F. J., Henden, A. A., Canzian, B., et al. 2000, ApJ, 528, 254

Waxman, E., \& Draine, B. T. 2000, ApJ, 537, 796

Weinberg, S. 1972, Gravitation and cosmology: Principles and applications of the general theory of relativity (Wilby, New York), 485

Zharikov, S. V., Sokolov, V. V., Baryshev, \& Yu. V. 1998, A\&A, 337, 356 